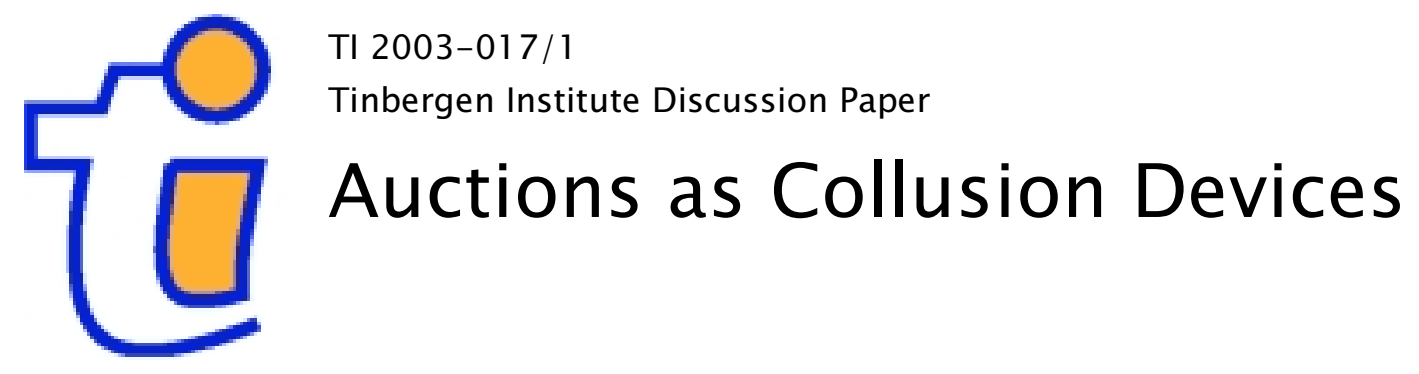

Maarten C.W. Janssen

Dept. of General Economics, Faculty of Economics, Erasmus University Rotterdam, and Tinbergen Institute. 


\section{Tinbergen Institute}

The Tinbergen Institute is the institute for economic research of the Erasmus Universiteit Rotterdam, Universiteit van Amsterdam, and Vrije Universiteit Amsterdam.

Tinbergen Institute Amsterdam

Roetersstraat 31

1018 WB Amsterdam

The Netherlands

Tel.: $\quad+31(0) 205513500$

Fax: $\quad+31(0) 205513555$

Tinbergen Institute Rotterdam

Burg. Oudlaan 50

3062 PA Rotterdam

The Netherlands

Tel.: $\quad+31(0) 104088900$

Fax: $\quad+31(0) 104089031$

Please send questions and/or remarks of nonscientific nature to driessen@tinbergen.nl.

Most TI discussion papers can be downloaded at http://www.tinbergen.nl. 


\title{
Auctions as Collusion Devices
}

\author{
Maarten C.W. Janssen*
}

This paper develops an economic argument relating auctions to high market prices. At the core of the argument is the claim that market competition and bidding in an auction should be analyzed as part of one game, where the pricing strategies in the market subgame depend on the bidding strategies during the auction.I show that the only equilibrium in the overall game that is consistent with the logic of forward induction is the one where firms bid an amount (almost) equal to the profits of the cooperative market outcome and follow a cooperative pricing strategy in the market game resulting in high prices.

Key words: auctions, market collusion

JEL-codes: L50

* Address for correspondence: prof. Maarten Janssen: Department of Economics, H0722, Postbox 1738, Erasmus University, 3000 DR Rotterdam, The Netherlands, Phone: 31-10-4082341, Fax: 31-10-4089149, E-mail: janssen@few.eur.nl. 


\section{Introduction}

One of the most debated questions concerning the recent wave of spectrum auctions held around the world is whether auctions give rise to higher prices in the market after the auction. Firms tend to stress that they have to recover the money they spend on obtaining a license and therefore tend to set higher prices when auction revenues are high. Economists tend to the view that payments during an auction should be considered as a sunk cost at the moment firms compete in the market place. According to the economic point of view, there is, or should not be, any relation between auction revenues and market prices (see, e.g., Binmore and Klemperer (2002)). Recent experiments show, however, that auctioning rights to compete in the market does lead to higher market prices (see, Offerman and Potters, 2000).

In this paper I want to develop an economic argument relating auctions to high market prices. At the core of the argument is the claim that market competition and bidding in an auction should be analyzed as part of one game. Pricing strategies in the market subgame can, in principle, depend on the bidding strategies during the auction. Vice-versa, the particular bidding behavior in an auction may be such that it signals future actions in the market game.

To make the argument more precise, I analyze a situation where there are $N$ bidders in the auction, a subset of which is competing in the market. The prize that the winners of the auction get is the right to play the market game. In its most simple form, the market competition game is analyzed as a coordination game in which firms can either follow a competitive strategy resulting in relatively low prices and profits or a cooperative (or tit-for-tat) strategy with relatively high prices and profits as a result if the players play the same strategy. As the market competition game is regarded as a simplified version of repeated interaction in the market, cooperative play can be supported as an equilibrium outcome.

More formally, the argument relating auctions to high market prices is translated in the claim that the only equilibrium in the overall game that is consistent with the logic of forward induction is the one where firms bid an amount (almost) equal to the profits of the cooperative market outcome and follow a cooperative strategy in the market game resulting in high prices. In other words, the auction solves the coordination game at the market level in favor of the high price (and high profit) equilibrium. Key steps in the proof 
of the argument are the following. First, ${ }^{1}$ the strategy "bid an amount in the auction game that is larger than the profits one can maximally achieve by choosing a competitive market strategy and choose a competitive market strategy" is dominated as it always leads to a negative profit. This in turn implies that if one of the firms that wins the auction has made a relatively high bid, the other firm can safely assume that this firm will choose to play cooperatively in the market game. Thus, the second step of the argument argues that strategies of the form "bid an amount $x$ in the auction game and choose a competitive market strategy whenever the other winning firm has bid an amount that is larger than the profits one can maximally achieve by choosing a competitive market strategy" is dominated by a similar strategy where cooperative play in the market game is recommended. These two steps together assure that if someone bids relatively high in the auction, firms play cooperatively in the market game. The last step in the argument shows that given this anticipation, competition between a large enough number of contestants in the auction assures that it is indeed optimal to bid higher than the profits one can maximally achieve by choosing a competitive market strategy.

An interesting side issue that comes out of the proof is that during the bidding stage in the game, firms may find it optimal to introduce a "jump bid" (see also Avery, 1998). The size of the jump is given by the difference between the maximal profits of following a competitive market strategy and the overall minimum pay-off winners of the auctions could make in the aftermarket. The jump is necessary to convince others to follow the cooperative strategy in the aftermarket.

Intuitively, what the above argument establishes is that by integrating the auction game and the market game into one larger game, auction expenditures are no longer sunk in the larger game. By looking at the market game separately, auction expenditures are indeed sunk, but at the auction stage they are not! Therefore auction expenditures may signal the intention of cooperative behaviour in the market game.

The more formal argument is related to the "burning money" argument in game theory (see, e.g., Van Damme, 1989, Osborne, 1990, Ben-Porath and Dekel, 1992 and Rubinstein, 1990). The basic idea in this literature is

\footnotetext{
${ }^{1}$ Before this step in the proof, a more straightforward point is made, namely that bids below the minimum pay-off of the market game are iteratively dominated by a slightly higher bid that is also below the minimum pay-off of the market game.
} 
that coordination problems like the ones in Battle-of-the-Sexes games can be resolved if one of two players before playing the game has the option to burn some money. The forward induction argument that is used in this literature is quite similar to the one applied in this paper. There are, however, some major differences. First, in an auction all players, and not just one, have the possibility of "burning money". Second, the competitive pressure present in auctions makes that in the resulting equilibrium, players $d o$ burn money, whereas the equilibrium that is selected in the burning money/Battleof -the-Sexes example, players do not burn money. The fact that nothing is burnt, leads to a fundamental issue regarding game theoretic modelling (see Osborne and Rubinstein, 1994, p. 113): One may always argue that players have the possibility of burning money, but as nothing is burnt it is difficult to perceive this as a signal as it is not clear whether the other player considered the possibility of burning money in the first place. My argument is not prone to this objection as the equilibrium that is chosen has players "burning money".

As discussed above, the standard economics argument seems to be that auction fees shoud not affect market prices. While making this point of view more precise in a section "popular objections to auctions", Binmore and Klemperer (2002, pp. C78) ${ }^{2}$ also say that

"and it is true that consumer prices can be affected [by auctions, MJ] (even by past lump-sum payments). For example, paying auction fees could potentially create 'focal points' that allow firms to coordinate tacitly on charging higher prices. Paying auction fees also makes firms poorer, so perhaps more willing to risk collusion, ... And an auction will in principle, select those firm that are better able to collude ... But all these effects seem small, and certainly avoidable with good competition policy."

This paper argues that there is another way in which auction fees may affect market prices, namely that the bids signal future decisions concerning prices. The argument is different from a focal point argument alluded to in the quote above as this paper's model has a unique equilibrium that is consistent with the forward induction logic. Focal points typically may be used when there are multiple equilibria. Collusion in the market place of the form described here may also be difficult to detect by competition authorities as no explicit communication is needed. Moreover, the firms may argue that

${ }^{2}$ Other interesting papers by Paul Klemperer on the European UMTS auctions, include Klemperer (2002a) and Klemperer (2002b). 
the auction has forced them to pay so much that if they don't cooperate in the market place, they will go bankrupt. If bankruptcy of crucial firms in an economy is a serious concern for competition authorities, there is not much the authorities can do after the auction has taken place. Of course, the authorities may threaten ex ante that if they find traces of collusive behavior, they will introduce severe punishments, but one may wonder whether this is a credible threat given the observation that ex post the authorties may not find it optimal to punish.

The paper is also related to recent literature on the interaction between auctions and aftermarkets. Jehiel and Moldovanu (1996a, 1996b, 2001) study the way externalities in the aftermarket have an impact on bidding behavior in the auction. They show that, depending on the specific context, standard properties of auctions do not hold when bidding firms also interact after the auction outcome has been established (see also, e.g., Das Varma, 2002). Signalling does not play a role in these papers. Signalling does play a role in Goeree (2002). In that paper players have private information that effects aftermarket competition. He shows that players may have an incentive to overstate their private information in an attempt to influence the behavior of competitors in the aftermarket. This paper, therefore, falls in the tradition of signalling models where actions (in this case, firms bidding behavior) may reveal a player's type. In contrast, I look at a situation where private information does not play a role and past actions signal future actions, instead of a player's private information.

The rest of the paper is organized as follows. Section 2 specifies the model. Section 3 contains the main proposition and its proof. Section 4 concludes with a discussion.

\section{The Model and Solution Concept}

There are $N$ firms. The game the firms play is a two-stage game. In the first stage the firms bid in an auction. The two firms with the highest bid continue to the second stage where they play a market competition game. If there is a tie for the first and/or second highest bid, a lottery will determine the ranking of the bids. The bids of the two players that continue to play the market competition game are denoted by $x_{1}$ and $x_{2}$, respectively, where $x_{1} \geq x_{2}$. Players only have to pay their bid in case they continue to the second stage of the game. For simplicity, but without loss of generality, I consider 
a first-price sealed-bid auction. For the formal part of the argument it is conventient to have a discrete strategy space and therefore, I assume firms can bid any amount $x=\epsilon, 2 \epsilon, 3 \epsilon, \ldots$ The grid $\epsilon$ measures the bidding increment and I assume that $\epsilon$ is small. ${ }^{3}$ In the second stage, the two winning firms play a market competition game. Firms can choose to play competitively (aggressively), denoted by $A$, or cooperatively, denoted by $C$. The $2 \times 2$ game is described in the matrix below

$$
\begin{gathered}
C \\
C \\
A
\end{gathered}\left(\begin{array}{cc}
a, a & c, d \\
d, c & b, b
\end{array}\right) \quad \text {, where } a>d \geq b>c>0 .
$$

So, the value $v$ of winning the auction is uncertain, and can be equal to $a, b, c$ or $d$. Note that the restrictions on the pay-off parameters imply that there are two symmetric pure-strategy equilibria: $(C, C)$ and $(A, A)$, where the first equilibrium Pareto-dominates the second. There are many ways one can interpret this game. One interpretation is that a static game (with quantities or other variables as strategic variables) is played that has multiple equilibria. Another interpretation is that the two players that win the auction play an infinitely repeated pricing game which has a collusive outcome $(C, C)$ and a competitive outcome $(A, A)$. The collusive outcome can be sustained through the use of trigger strategies. ${ }^{4}$ In line with this second interpretation, I denote the action of player $i$ in the market game by $p_{i}$. The overall strategy of player $i$ is then denoted by $\left(x_{i}, p_{i}\left(x_{1}, x_{2}\right)\right)$, i.e. (and this is important for the argument that follows) the market behavior of the players in the second stage of the game may depend on the winning bids in the auction.

It is clear that any type of market behavior can be part of a subgame perfect equilibrium. Underlying the notion subgame perfection is the view that deviations from a proposed equilibrium strategy are considered mistakes which are not informative of future behavior (cf., Selten, 1975). The only requirement it imposes is that the strategies in the market game form an equilibrium in the market subgame and in our case there are two equilibria. ${ }^{5}$

\footnotetext{
${ }^{3}$ Note that in most auction designs a bid increment of some kind is implemented.

${ }^{4}$ For the argument that follows it is not necessary that $d \geq b$. The assumption is made to avoid writing $\max (b, d)$ each time. Moreover, in line with the collusion and trigger strategy interpretation, the assumption $d \geq b$ is more natural than the reverse.

${ }^{5}$ Note that in the present context the notion of backward induction formalises the standard economists' point of view of "auction revenues are sunk cost".
} 
The argument I made in the Introduction, namely that a deviation of a proposed equilibrium bid in the auction game should not be interpreted as a random mistake, but rather as a signal of future actions in the market game is more in line with the notion of forward induction (see, e.g., Kohlberg and Mertens, 1986, Van Damme 1989, and others). An equilibrium is consistent with forward induction if it is not the case that some player, by deviating at a node along the equilibrium path, can ensure that a proper subgame is reached where all solutions but one give the player strictly less than the equilibrium pay-off, and where exactly one solution gives the player strictly more. Underlying the notion of forward induction is the idea that deviations from a proposed equilibrium should be interpreted as signals of future actions, if possible. The solution concept underlying the argument above is iterative elimination of weakly dominated strategies (IEDS).

\section{Analysis}

In this section, I prove the main result of the paper. The result says that provided the number of competing firms in the auction is large enough, the forward induction argument selects only one of the subgame perfect equilibria. The equilibrium that is selected has firms colluding in the market game. Moreover, during the auction phase firms "burn" all their future profits, i.e., their bids are close to the profits obtained in the market game.

Theorem 1 For any $N \geq \max \left\{\frac{2(a-c+2 \epsilon)}{(a-d-\epsilon)}, 4\right\}$ the unique equilibrium that is consistent with forward induction has $x_{i} \in[a-2 \epsilon, a)$ and $p_{i}\left(x_{1}, x_{2}\right)=C$, $i=1, \ldots, N$.

Proof. The proof eliminates sets of strategies in five consecutive stages. First, define $K$ as the largest integer such that $(K+1) \epsilon<c$.

Step $i$ : Fix an integer $k$ with $0 \leq k \leq K-1$. Given that players' strategies are restricted to $x_{i} \geq k \epsilon$, any strategy $\left(x_{i}, p_{i}\left(x_{1}, x_{2}\right)\right)$ with $x_{i}=k \epsilon$ is iteratively dominated by $\left(\widetilde{x}_{i}, \widetilde{p}_{i}\left(x_{1}, x_{2}\right)\right)$ with $\widetilde{x}_{i}=(k+1) \epsilon$ and $\widetilde{p}_{i}\left(x_{1}, x_{2}\right)=$ $p_{i}\left(x_{1}, x_{2}\right)$.

The argument here is quite similar to the conventional elimination of dominated strategies in a first-price sealed-bid auction. The pay-offs the two strategies yield in the second stage is the same, because $\widetilde{p}_{i}\left(x_{1}, x_{2}\right)=$ 
$p_{i}\left(x_{1}, x_{2}\right)$, and at least equal to $c$. Moreover, the overall pay-off of both strategies is always positive as $\widetilde{x}_{i} \leq c-\epsilon$. The firms thus try to outbid each other, which is what drives the bids up. The last step of the this stage of the elimination procedure is the most stringent and gives a good idea about the previous steps. So, let us briefly consider the argument for the $K$ 'th step. In the $K$ 'th step I have to argue that any strategy $\left(x_{i}, p_{i}\left(x_{1}, x_{2}\right)\right)$ with $x_{i}=(K-1) \epsilon$ is iteratively dominated by $\left(\widetilde{x}_{i}, \widetilde{p}_{i}\left(x_{1}, x_{2}\right)\right)$ with $\widetilde{x}_{i}=K \epsilon$ and $\tilde{p}_{i}\left(x_{1}, x_{2}\right)=p_{i}\left(x_{1}, x_{2}\right)$. There are three possible situations to consider: Either $x_{2}>K \epsilon$, or $x_{2}=K \epsilon$, or $x_{2}=(K-1) \epsilon$. In the first case, both classes of strategies (those with $x_{i}=(K-1) \epsilon$ and those with $x_{i}=K \epsilon$ ) under consideration yield a pay-off of 0 . In the second case, all strategies with $x_{i}=(K-1) \epsilon$ yield a pay-off of 0 , whereas strategies with $x_{i}=K \epsilon$ yield a positive expected pay-off. In the third case, the pay-off of strategies in the class with $x_{i}=(K-1) \epsilon$ yield a pay-off of at most $2(v-(K-1) \epsilon) / N$, where $v$ equals $a, b, c$ or $d$. The pay-off of strategies in the class with $x_{i}=K \epsilon$ yield a pay-off of $v-K \epsilon$. This latter expression is larger than the first expression for any value of $v$, if it is larger for $v=c$. As $(K-1) \epsilon<c-2 \epsilon$, this is the case when $4 \epsilon / N \leq \epsilon$, or $N \geq 4$.

Step $i i$ : Any strategy $\left(x_{i}, p_{i}\left(x_{1}, x_{2}\right)\right)$ with $x_{i} \geq d$ and $p_{i}\left(x_{1}, x_{2}\right)=A$ whenever $x_{i} \geq x_{2}$ is weakly dominated by $\left(\widetilde{x}_{i}, \widetilde{p}_{i}\left(x_{1}, x_{2}\right)\right)$ with $\widetilde{x}_{i}<d$ and $\tilde{p}_{i}\left(x_{1}, x_{2}\right)=p_{i}\left(x_{1}, x_{2}\right)$.

To prove this claim, I will denote the first strategy by $s_{1}$ and the second one by $s_{2}$. When player $i$ sets strategy $s_{1}$, her pay-off is either 0 or negative. I will show that by choosing strategy $s_{2}$, she can never do worse and sometimes better. There are three possibilities: $x_{2}>x_{i}, \widetilde{x}_{i} \leq x_{2} \leq x_{i}, \widetilde{x}_{i}>x_{2}$. In the first case, both $s_{1}$ and $s_{2}$ yield a pay-off of 0 . In the second case, $\pi_{i}\left(s_{1}, s_{-i}\right) \leq 0 \leq \pi_{i}\left(s_{2}, s_{-i}\right)$. In the third case, $\pi_{i}\left(s_{1}, s_{-i}\right)<0<\pi_{i}\left(s_{2}, s_{-i}\right)$. Thus, $s_{2}$ weakly dominates $s_{1}$.

Step iii: Any strategy $\left(x_{i}, p_{i}\left(x_{1}, x_{2}\right)\right)$ that assigns $p_{i}\left(x_{1}, x_{2}\right)=A$ for some value of $x_{1} \geq d$ is weakly dominated by $\left(\widetilde{x}_{i}, \widetilde{p}_{i}\left(x_{1}, x_{2}\right)\right)$ with $\widetilde{x}_{i}=x_{i}$ and $\tilde{p}_{i}\left(x_{1}, x_{2}\right)=C$ whenever $x_{1} \geq d$ and otherwise $\widetilde{p}_{i}\left(x_{1}, x_{2}\right)=p_{i}\left(x_{1}, x_{2}\right)$.

To prove this claim, note that at this stage of the argument all strategies $\left(x_{i}, p_{i}\left(x_{1}, x_{2}\right)\right)$ with $x_{i} \geq d$ that survived IEDS have $p_{i}\left(x_{1}, x_{2}\right)=C$ because of step (ii). There are two cases then to consider: $x_{i}<x_{2}$ and $x_{i}=x_{2}{ }^{6}$ In the first case, both strategies yield a pay-off of 0 . In the second case, let us denote by $m$ the number of players with a bid equal to $x_{i}$. There are

\footnotetext{
${ }^{6}$ Note that the case $x_{i}>x_{2}$ is covered by $(i i)$ above as it implies that $x_{i}=x_{1} \geq d$.
} 
two subcases: $x_{1}$ equals a value larger than or equal to $d$ to which the first strategy assigns $p_{i}\left(x_{1}, x_{2}\right)=A$ and all other values of $x_{1}$ including $x_{1}<d$. In the first subcase, the overall pay-off of the first strategy is $(d-x) / m$, whereas the pay-off of the second strategy is $(a-x) / m$. In the second subcase, the actions prescribed by both strategies are identical and, therefore, the pay-offs are equal.

Steps $i-i i i$ together assure that if one player bids an amount larger than or equal to $d$ in the auction, both players proceeding to the second stage of the game will choose to play cooperatively. The next step argues that all strategies that prescribe players to bid less than $d$ in the auction are iteratively dominated. To this end let us denote by $\widehat{S}^{C}(0)$ the set of strategies $\left\{\left(x_{i}, p_{i}\left(x_{1}, x_{2}\right)\right) \mid x_{i}>c-2 \epsilon\right.$ and $p_{i}\left(x_{1}, x_{2}\right)=C$ if $\left.x_{1} \geq d\right\}$. Note that this class leaves the second stage action unspecified whenever $x_{1}<$ d. Let us also define $\widetilde{S}^{C}(0)$ as the subset of $\widehat{S}^{C}(0)$ with the lowest bid $x_{i}$, i.e., $\widetilde{S}^{C}(0) \equiv\left\{\left(x_{i}, p_{i}\left(x_{1}, x_{2}\right)\right) \mid c-2 \epsilon<x_{i}<c-\epsilon\right.$ and $p_{i}\left(x_{1}, x_{2}\right)=C$ if $\left.x_{1} \geq d\right\}$. The lowest bid itself in $\widetilde{S}^{C}(0)$ is denoted by $\widehat{x}(0)$. Using these two notions, we can define $\widehat{S}^{C}(1) \equiv \widehat{S}^{C}(0) \backslash \widetilde{S}^{C}(0)$ and similarly to defining $\widetilde{S}^{C}(0)$, one can define $\widetilde{S}^{C}(1)$ as the subset of $\widehat{S}^{C}(1)$ with the lowest bid $x_{i}$, i.e., $\widetilde{S}^{C}(1) \equiv\left\{\left(x_{i}, p_{i}\left(x_{1}, x_{2}\right)\right) \mid c-\epsilon<x_{i}<c\right.$ and $p_{i}\left(x_{1}, x_{2}\right)=C$ if $\left.x_{1} \geq d\right\}$. Proceeding iteratively, I define for all $k>1, \widehat{S}^{C}(k) \equiv \widehat{S}^{C}(k-1) \backslash \widetilde{S}^{C}(k-1)$ and $\widetilde{S}^{C}(k)$ as the subset of $\widehat{S}^{C}(k)$ with the lowest bid $x_{i}$. In each round the lowest bid itself in $\widetilde{S}^{C}(k)$ is denoted by $\widehat{x}(k)$. Finally, I define $K^{C}$ as the number of steps $\epsilon$ that is needed to reach the interval $[d-\epsilon, d]$ from the interval $[c-2 \epsilon, c-\epsilon]$.

Step iv: Fix a $1 \leq k \leq K^{C}$ and $\widehat{S}^{C}(k)$. Given that players' strategies are restricted to $\widehat{S}^{C}(k)$, all strategies in $\widetilde{S}^{C}(k)$ are weakly dominated by the strategy $\left(x_{i}, p_{i}\left(x_{1}, x_{2}\right)\right)$ with $d \leq x_{i}<d+\epsilon$ and $p_{i}\left(x_{1}, x_{2}\right)=C$ for all pairs $\left(x_{1}, x_{2}\right)$.

To prove this step, let us call the dominating strategy $s_{4}$. For each $k \geq 0$ there are three situations to consider. Either $x_{2} \geq d+\epsilon$, or $\widehat{x}(k)<x_{2}<d+\epsilon$, or $\widehat{x}(k)=x_{2}$. In the first case, all strategies in $\widetilde{S}^{C}(k)$ as well as strategy $s_{4}$ itself yield a pay-off of 0 . In the second case, all strategies in $\widetilde{S}^{C}(k)$ yield a pay-off of 0 , whereas strategy $s_{4}$ yields a pay-off larger than $a-d-\epsilon$ due to the fact that step (iii) implies that if someone bids an amount higher than $d$, players play $C$. In the third case, the pay-off of strategy $s_{4}$ is still larger than $a-d-\epsilon$, whereas the pay-off of choosing a strategy in $\widetilde{S}^{C}(k)$ cannot be larger than $2(a-c+2 \epsilon) / N$. When $N$ satisfies the condition mentioned in the Theorem, the first pay-off is not smaller than the second. 
Steps $i-i v$ imply that all strategies with bids $x_{i}<d$ are iteratively eliminated. The last step of the argument then is again a conventional auction type of argument. To this end, define $K^{d}$ as the smallest integer such that $K^{d} \epsilon>d$ and $K^{A}$ as the largest integer such that $\left(K^{A}+1\right) \epsilon<a$.

Step $v$ : Fix an integer $k$ with $K^{d} \leq k \leq K^{A}-1$. Given that players bidding strategies are restricted to $x_{i} \geq k \epsilon$, any strategy $\left(x_{i}, p_{i}\left(x_{1}, x_{2}\right)\right)$ with $x_{i}=k \epsilon$ and $p_{i}\left(x_{1}, x_{2}\right)=C$ for all pairs $\left(x_{1}, x_{2}\right)$ is iteratively dominated by $\left(\widetilde{x}_{i}, \widetilde{p}_{i}\left(x_{1}, x_{2}\right)\right)$ with $x_{i}=(k+1) \epsilon$ and $\widetilde{p}_{i}\left(x_{1}, x_{2}\right)=p_{i}\left(x_{1}, x_{2}\right)$.

This last step of the argument is, again, just the conventional argument of elimination of dominated strategies in a first price sealed-bid auction. Given steps (ii)-(iv) firms always play cooperatively in the market game, which guarantees a pay-off of $a$ of winning the auction. Firms would like therefore, to outbid each other, which drives the bids in the auction up. The details of the argument are similar to the argument made in step $(i)$ and are, therefore, omitted.

Step (iv) of the proof highlights the use of a "jump bid". ${ }^{7}$ Given the earlier steps of the proof, a bidder can only guarantee himself the highest possible continuation pay-off in the aftermarket, if he chooses a bid that is higher than the maximal pay-off of $d$ one could get by following market strategy $A$. Up to that moment in the auction (proof) only bids smaller than $c$ are eliminated. The size of the jump bid is thus at least equal to $d-c$.

One issue that remains to be discussed is why the argument only works when the number of firms is larger than a specific lower bound on the number of firms participating in the auction. The reason is the following. There is a possibility that bidding stops at the moment all bidders bid an amount $x_{i}$ close to $c-\epsilon$. The two firms that are randomly selected face a coordination problem in the market game: both playing cooperatively and both playing aggresively are both Nash equilibria. Even though there is no specific reason to do so, it may thus happen that both firms coordinate on playing cooperatively. The total pay-off for the two firms of following this strategy is then smaller than $a-c+2 \epsilon$. The chance of being selected is $2 / N$. Note that the expected pay-off decreases in $N$ as the chance of being selected in the lottery decreases. Each firm then faces the following decision problem: being satisfied with this chance of getting a relatively large pay-off or "jump

\footnotetext{
${ }^{7}$ Of course, jump bids taking literally cannot take place in a sealed-bid auction. However, a similar analysis applies to a multi-unit ascending auction.
} 
bidding" to a bid larger than $d$, which guarantees a pay-off of $a$ in the market game. For "jump bidding" to be profitable, $N$ has to be relatively large.

An important question, of course, is to what extent the result of the paper holds true in more general settings. Different, interesting, extensions can be considered: $(i)$ what if more than two licenses are auctioned off? $(i i)$ what if firms receive different pay-offs at the market stage depending on who wins the auction? Here, I will briefly indicate to what extent the result obtained here can be generalized.

If instead of two, $k>2$ licenses are offered for sale, the result is affected only in a quantative sense. In order to briefly discuss the implications of this generalization, we first need to generalize the market stage pay-offs to the case where $k$ firms compete. The pay-offs when everyone behaves cooperatively or aggressively do not need to be modified. When $n<k$ players play cooperatively and the remaining $k-n$ players play aggressively, one may denote the pay-off to the aggressors and cooperators by $d_{n}$ and $c_{n}$, respectively. It is natural to assume that $d_{n}<d_{n+1}$ and $c_{n}<c_{n+1}$, i.e., the more cooperators, the higher the pay-offs to both cooperators and aggressors. The condition for the Theorem to hold changes to $N>\max \left\{\frac{k\left(a-c_{1}-\epsilon\right)}{\left(a-d_{n-1}-\epsilon\right)}, 2 k\right\}$. The fact that the condition on $N$ becomes tighter is understood if one realizes the fact that with more licenses being auctioned, the chance of getting one of them increases if players bid relatively low amounts. The "jump" that has to be made in order to signal future cooperative behaviour remains the same, however, and therefore, the cost of making such a "jump bid". The main argument, however, remains the same: if someone bids an amount during the auction that he cannot hope to receive in the market game by playing aggressively, i.e., if he bids more than $d_{n-1}$, then he signals future cooperative behavior. Also, during the auction stage firms compete away their future profits, like in the Theorem stated in the previous section.

Another possible extension is to allow for the possibility of having playerdependent pay-offs in the market game: for any $v=a, b, c$ or $d$, we may write $v_{i}(i, j)$, which makes clear that the pay-offs of cooperating or competing in the market place may depend on the identity of the pair of winning firms $(i$ and $j$ ) and on the identity of each of them. New phenomena may arise in this case such as coordination issues, if, for example, one firm expects high pay-offs when playing the market game with a particular other firm, but not with another. If the differences between the firms are not too large, however, (in a sense to be made precise) we may expect the core of the Theorem to hold true. 


\section{Discussion and Conclusion}

In this paper, I have shown in the context of a simple model how auctions may lead to high prices in the after-auction market. The main idea is that by bidding more than the profits a firm could possibly make by playing a competitive strategy in the market game, a firm signals that he will act cooperatively in the market game. Other firms pick up this signal and play cooperatively as well if they take part in the market game. As during the auction, firms compete to get a license to operate in the after-auction market, firms outbid each other during the auction game. Thus, all firms bid more than they possibly could make by competing in the after-auction market.

It is important to note that some conditions are necessary to make the argument work: $(i)$ there should not be too much uncertainty about future market pay-offs, (ii) equilibrium behavior in the market should be indetermined in the sense that multiple equilibria in the market game exists, (iii) the winning bids should be publicly observable, and ( $i v)$ the number of contestants in the auction should be relatively large. I will briefly comment on the first three points below; the fourth issue has already been discussed in Section 3 .

Concerning uncertainty, the argument made allows for some form of payoff uncertainty as long as the maximum pay-off from competing aggressively in the market stage is smaller than the minimum pay-off of all firms colluding in the market place. In case there is too much uncertainty about future payoffs, auction fees cannot be interpreted as a signal of future market stage behaviour. In the case of the European UMTS-auctions, one may argue that they were held at such an early point in time that the perspective on profits to be gained was highly uncertain. If this is so, the above argument does not apply.

When there is a unique equilibrium in the market stage, signalling future behavior does not make much sense as future behavior is fully determined by the market constellation itself. Hence, a necessary condition for our argument to work is the existence of multiple market equilibria. Finally, when the winning bids are not made public, firms cannot condition their market behavior on these winning bids. Accordingly, the firms cannot use their bids to signal future intentions in this case.

There are several interesting policy issues concerning auction design that come out of this paper. A first issue is the timing aspect: when is the time ripe to hold an auction? One may argue that auctions should be held when 
firms are quite certain about the market potential of the objects (frequencies) that are auctioned as this will diminish a firm's fear of paying too much and it may boost competition during the auction. This paper argues, however, that there may be a countervailing argument as well: waiting to reduce the amount of uncertainty may make it easier for firms to signal collusive intentions at the market stage.

A second issue arises with respect to the number of competitors. Standard auction theory would say that the more competitors, the better the outcome to be expected from the perspective of the auctioneer. Also, in this case the paper offers a warning: it may be more profitable to signal the intention to collude in the market place when the number of contestants in the auction is large. So, auction revenues may indeed be high with a large number of contestants, but welfare generated in the aftermarket may be low.

A third issue arises with respect to the optimal choice of bid increment. It is easy to see that the lower bound on the number of firms stated in the Theorem is increasing in the bid increment $\epsilon$. This means that by choosing a larger bid increment, the auctioneer (government authorities) may try to prevent signalling in the sense discussed in this paper.

Finally, it is interesting to observe that by announcing the winning bids, the government may facilitate collusion in the market place in the sense discussed in this paper. If only the identity of the winning bidders is revealed, but not their bids, firms cannot directly infer what the other firm has paid and therefore they cannot condition market behavior on the bids. In this case, the argument developped in this paper breaks down.

\section{References}

Ben-Porath, E. and E. Dekel. 1992. Signalling Future Actions and the Potential for Sacrifice. Journal of Economic Theory 57, p. 36-51.

Binmore, K. and P. Klemperer. 2002. The Biggest Auction Ever: The Sale of the British 3G Telecom Licenses. Economic Journal 112: C-74-96.

Goeree, J. 2002. Bidding for the Future. Journal of Economic Theory (forthcoming).

Jehiel, P. and B. Moldovanu. 1996a. Strategic Nonparticipation. Rand Journal of Economics 27, 84-98.

Jehiel, P. and B. Moldovanu. 1996b. How (not) to sell Nuclear Weapons. American Economic Review 86, 814-29. 
Jehiel, P. and B. Moldovanu. 2001.Auctions with Downstream Interaction among Buyers. Rand Journal of Economics 31, 768-91.

Klemperer (2002a) What really matters in auction design. Journal of Economic Perspectives 16, p. 169-189.

Klemperer (2002b) How not to run auctions: the European 3G mobile telecom auctions. European Economic Review (forthcoming).

Kohlberg, E. and J.-F. Mertens. 1986. On the Strategic Stability of Equilibria. Econometrica 54, p. 1003-1037.

Offerman, Th. and J. Potters. 2000. Does Auctioning of Entry Licenses affect Consumer Prices? Tinbergen Institute Discussion Paper 00-046/1.

Osborne, M. 1990. Signalling, Forward Induction and Stability in Finitely Repeated Games. Journal of Economic Theory 50, p. 22-36.

Osborne, M. 1990. and A. Rubsinstein, A. 1994. A Course in Game Theory. MIT Press.

Rubsinstein, A. 1991. Comments on the Interpretation of Game Theory. Econometrica 59, p. 909-924.

Selten, R. 1975. Reexamination of the Perfectness concept for equilibrium points in extensive games. International Journal of Game Theory 4, 25-55.

Van Damme, E. 1989. Stable Equilibria and Forward Induction. Journal of Economic Theory 48, p. 476-496. 